\title{
Ocean gravity wave phase velocity detection by HFSWR
}

\author{
Lun $\mathrm{Li}^{\mathrm{a})}$, Xiongbin Wu${ }^{\mathrm{b})}$, Xing'an Xu, Bin Liu, Chao Long, \\ Zhiben Shen, and Xiaofeng Chen
}

Institute of Electronic Information, Wuhan University, Wuhan, China, 430072

a) lilunhp@163.com

b)xbwu@whu.edu.cn

Abstract: The formula of inversion ocean gravity wave phase velocity from high frequency surface wave radar (HFSWR) sea echo is derived. By field data analysis at deep water condition, the ocean gravity wave phase velocity is verified by comparison with theoretical velocity based on deep water dispersion relationship.

Keywords: ocean gravity wave phase velocity, high frequency surface wave radar, inversion

Classification: Electron devices, circuits, and systems

\section{References}

[1] L. Barnes, "HF Radar-The Key to Efficient Wide Area Maritime Surveillance," EEZ Technology, Edition 3, pp. 115-118, ICG Publishing LTD, 1998.

[2] D. E. Barrick, "30 Years of CMTC and CODAR," Proc. IEEE/OES/ CMTC Ninth Working Conference on Current Measurement Technology, pp. 131-136, 2008.

[3] L. R. Wyatt, J. J. Green, A. Middleditch, M. D. Moorhead, J. Howarth, M. Holt, and S. Keogh, "Operational Wave, Current, and Wind Measurements With the Pisces HF Radar," IEEE J. Ocean. Eng., vol. 31, no. 4, pp. 819-834, 2006.

[4] H.-H. Essen, K.-W. Gurgel, and T. Schlick, "On the accuracy of current measurements by means of HF radar," IEEE J. Ocean. Eng., vol. 25, no. 4, pp. 472-480, 2000.

[5] W. Xiongbin, Y. Shaolin, C. Feng, W. Shicai, Y. Zijie, W. Biyang, S. Zhenhua, T. Jiansheng, H. Jiechang, and K. Hengyu, "Ocean Surface Currents Detection at the Eastern China Sea by HF Surface Wave Radar," Chinese J. Geophysics, vol. 46, no. 3, pp. 340-346, 2003.

[6] D. E. Barrick and B. J. Lipa, "The second-order shallow water hydrodynamic coupling coefficient in interpretation of HF radar sea echo," IEEE J. Ocean. Eng., vol. 11, pp. 310-315, 1986.

[7] B. Lipa, B. Nyden, D. Barrick, and J. Kohut, "HF Radar Sea-echo from Shallow Water," Sensor, vol. 8, pp. 4611-4635, 2008.

[8] D. E. Barrick, "First-order theory and analysis of MF/HF/ VHF scatter from the sea," IEEE Trans. Antennas Propag., vol. 20, no. 1, pp. 2-10, 1972.

[9] R. Schmidt, "Multiple emitter location and signal parameter estimation," IEEE Trans. Antennas Propag., vol. 24, no. 3, pp. 276-280, 1986.

[10] J. Chen, "Research of experimentation and algorithm on ocean current extraction by high frequency ground wave radar," Wuhan University, 
pp. 20-21, 2005.

[11] W. Xiongbin, C. Feng, Y. Zijie, and K. Hengyu, "Broad Beam HFSWR Array Calibration Using Sea Echoes," International Conference on Radar, pp. 1-3, Oct. 2006.

\section{Introduction}

High frequency surface wave radar (HFSWR) takes advantage of the low attenuation characteristic of high frequency electromagnetic waves $(3 \mathrm{MHz}$ to $30 \mathrm{MHz}$ ) propagating across the ocean surface to measure the ocean surface current, wind and wave [1]. Current is measured by Doppler principle based on the Bragg resonance between radio wave and ocean gravity waves with half wavelength of radio wave moving directly towards and away from radar $[2$, $3,4,5]$.

Till now ocean surface current inversion from the HFSWR all assume deep water condition and the Bragg ocean gravity wave phase velocity is independent of water depth. But in fact, there is shallow water which may cause uncertain about the ocean gravity wave phase velocity, especially at the near shore area closely related with people's lives. This will influence the accuracy of ocean surface dynamic parameters inversion from HFSWR $[6,7]$.

In this paper, ocean surface gravity wave phase velocity inversion method from HFSWR sea echo is provided. The phase velocity calculation formula is derived with the positive and negative peek of the first order Doppler spectra. The algorithm diagram of gravity wave phase velocity is also introduced. In order to test the advanced method, field experiment data is analyzed.

\section{Principle}

Barrick derived the first-order sea echo radar cross section by perturbation method which lay the foundation for HF radio wave ocean surface current detection [8]. The formula can be expressed as

$$
\sigma^{(1)}(\omega)=2^{6} \pi k_{0}^{4} \sum_{m= \pm 1} S\left(-2 m \vec{k}_{0}\right) \delta\left(\omega-m \omega_{\mathrm{B}}\right)
$$

where $\omega$ is the Doppler frequency, $\omega_{\mathrm{B}}$ is the resonant Bragg circular frequency, $S\left(-2 m \vec{k}_{0}\right)$ is the directional wave spectrum of ocean wave $-2 m \vec{k}_{0}, \vec{k}_{0}$ is the wave vector of incident radio wave, $m= \pm 1$ denotes the two wave vector moving towards and away from the radar. The $\delta$ function describes a pair of distinct peaks located on either side of the $0 \mathrm{~Hz}$ line.

In the formula (1) $\omega_{\mathrm{B}}$ is caused by ocean gravity wave phase velocity, and the current velocity can also cause Doppler shift, so the Doppler frequency can be denoted as

$$
\left\{\begin{array}{l}
f_{+}=f_{B}+f_{r} \\
f_{-}=-f_{B}+f_{r}
\end{array}\right.
$$

where $f_{+}$and $f_{-}$are the Doppler frequency of positive and negative firstorder spectra respectively, $f_{B}$ is Bragg Doppler frequency and $f_{B}=\frac{\omega_{B}}{2 \pi}, f_{r}$ 
is the Doppler shift caused by current. And we have

$$
\left\{\begin{array}{c}
f_{B}=\frac{2 V_{P}}{\lambda_{0}} \\
f_{r}=\frac{2 V_{r}}{\lambda_{0}}
\end{array}\right.
$$

where $V_{P}$ is ocean gravity wave velocity, $V_{r}$ is ocean radial current and $\lambda_{0}$ is radio wavelength.

In deep water, the dispersion relationship for gravity wave is $\omega^{2}=g k$, and phase velocity $V_{P}=\frac{\omega}{k}=\sqrt{\frac{g}{k}}$, where $\mathrm{g}$ is gravity acceleration, $k$ is gravity wave number, so we know that when ocean gravity wave number is constant, the phase velocity is invariable, and the Doppler frequency caused by gravity wave is invariable. But in shallow water, the phase velocity is a function of water depth, which is not suitable for current inversion with the popular algorithm. In this paper we process $(2)$ by subtraction and obtain the $f_{B}$

$$
f_{B}=\frac{f_{+}-f_{-}}{2}
$$

Simultaneous equation (3) and (4), we can obtain the ocean gravity wave phase velocity

$$
V_{P}=\frac{\left(f_{+}-f_{-}\right) \lambda_{0}}{4}
$$

\section{Phase velocity inversion from broad beam HFSWR}

\subsection{Inversion method}

The currently HFSWR in the world such as SeaSonde, Wera (Wellen Radar) and OSMAR (Ocean State Monitoring and Analysis Radar) are all broad beam HF radars. This kind of HF radars have one transmitting antenna with a broad wave beam up to $120^{\circ}$ and with 3 to 16 antennas consisting as a receiving array the signal direction of arrival can be estimated, so the firstorder spectra of sea echo is compose of sea echo from broad directions. To the broaden first-order spectra we cannot use (6) to calculate ocean gravity wave velocity directly, and we should get the Doppler spectra points at the same direction first. Here we can use spectral space estimation method to estimate signal direction and Fig. 1 shows the basic flow chart of phase velocity inversion from broad beam HFSWR.

\subsection{Direction estimation}

To the broad beam HFSWR, MUSIC (Multiple Signal Characteristic) algorithm is widely used for signal direction of arrival estimation. The algorithm is super-resolution, small amount of calculation and fast speed [9]. Assuming that the receiving antenna array has $\mathrm{N}$ elements and the coordinate denotes as $\left(x_{i}, y_{i}\right), i=1,2, \cdots \cdots N$, the directions of narrow band signal are $\theta_{1}, \theta_{2}, \cdots \cdots, \theta_{k}$, then the signal model of receiving array is

$$
\mathbf{X}(t)=\mathbf{A}(\theta) \mathbf{S}(t)+\mathbf{N}(t)
$$




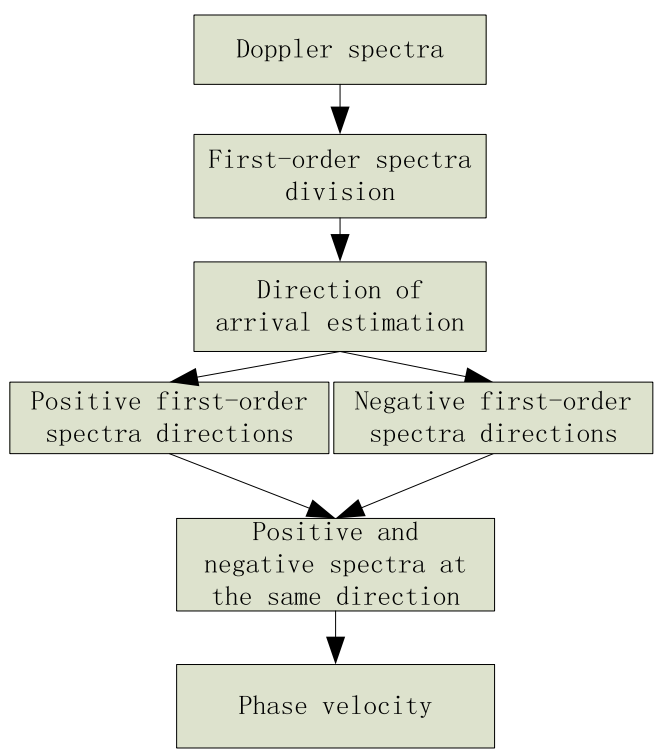

Fig. 1. The flow chart of phase velocity inversion from broad beam HFSWR

where $\mathbf{A}=\left[\begin{array}{llll}\mathbf{a}\left(\theta_{1}\right) & \mathbf{a}\left(\theta_{2}\right) & \cdots & \mathbf{a}\left(\theta_{k}\right)\end{array}\right], \tau_{i}=\left[x_{i}, y_{i}\right][\sin (\theta), \cos (\theta)]^{T}$ and $\mathbf{a}\left(\theta_{i}\right)=$ $\left[1, \exp \left(-j \frac{2 \pi}{\lambda} \tau_{2}\right), \cdots, \exp \left(-j \frac{2 \pi}{\lambda} \tau_{N}\right)\right]^{T}, \lambda$ denotes the wavelength of radio wave, $\mathbf{N}(t)$ and $\mathbf{S}(t)$ are the observation noise and signal. And the estimation of covariance matrix $\mathbf{R}_{x x}$ is

$$
\mathbf{R}_{x x}=E\left[\mathbf{X}(t) \mathbf{X}^{H}(t)\right]=\mathbf{A} \mathbf{R}_{s s} \mathbf{A}^{H}+\sigma^{2} \mathbf{I}
$$

By eigenvalue decomposition (EVD) the observation space can be partitioned into a signal subspace $\mathbf{E}_{S}$ and a noise subspace $\mathbf{E}_{N}$. And the two subspace is orthogonal, so we can obtain the MUSIC spectra [9]

$$
P_{\text {MUSIC }}=\frac{1}{\mathbf{A}^{\mathbf{H}} \mathbf{E}_{N} \mathbf{E}_{N}^{H} \mathbf{A}}
$$

In formula (8), the determination of signal subspace and noise subspace needs estimate the signal number and the general methods are gerschgorin disk criterion method, eigenvalue gradient method and so on. In this paper, the eigenvalue gradient method is used, which can refer to reference [10].

\section{Data analysis}

\subsection{Radar system}

The HFSWR system OSMAR071 is located at Longhai, Fujian Province of China Sea. The system is consists of transmitter, receiver, antenna system, display and storage system. Fig. 2 (a) shows the hardware structure. OSMAR071 applies the Frequency Modulated Interrupted Continuous Wave (FMICW) waveform with $30 \mathrm{kHz}$ bandwidth and $5 \mathrm{~km}$ distance resolution. A three-element Yagi-Uda antenna serves as transmitting antenna and an 8-element non-linear receiving array as the radar antenna filed. Array amplitude and phase correction is obtained by real-time software calibration [11]. 


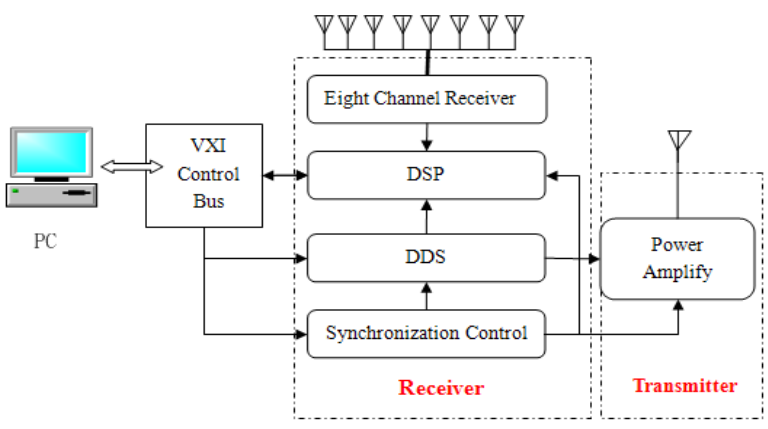

(a)

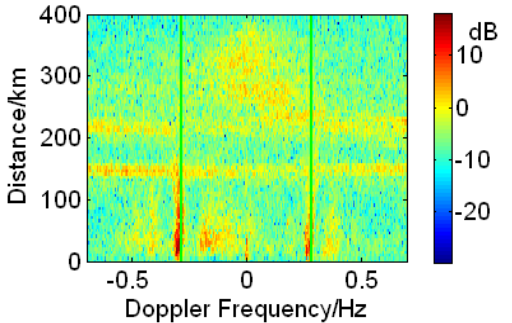

(b)

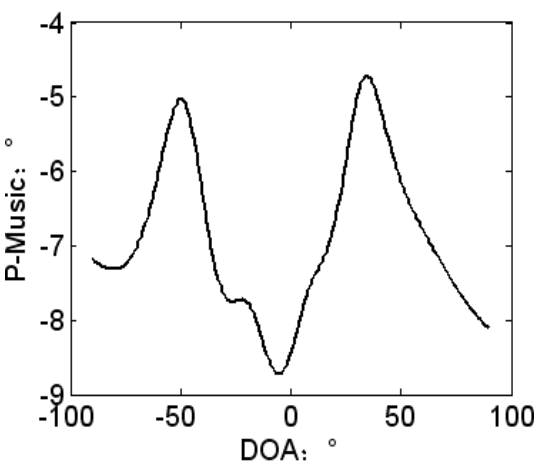

(d)

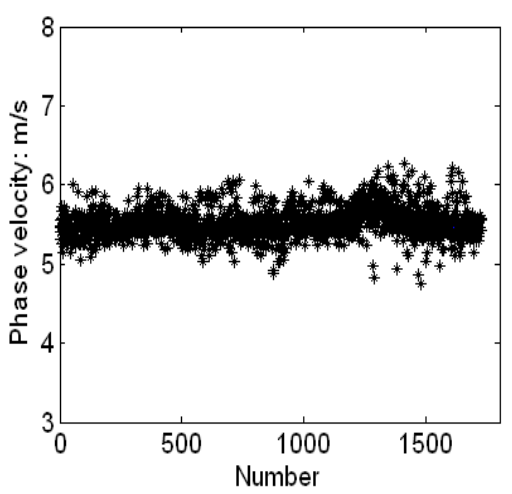

(f)

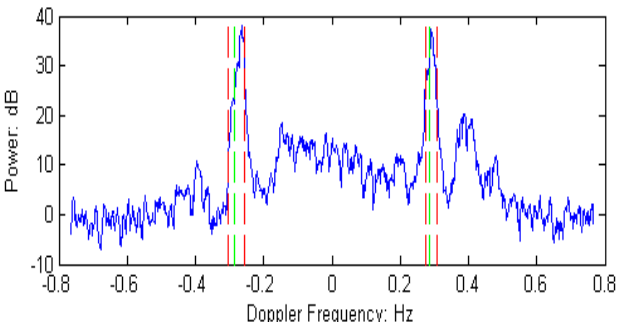

(c)

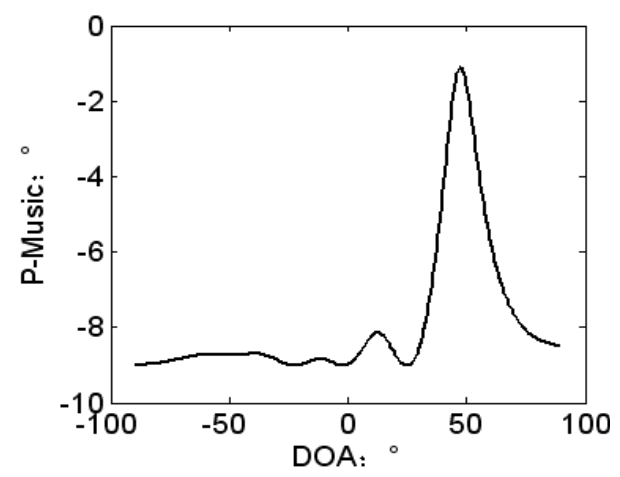

(e)

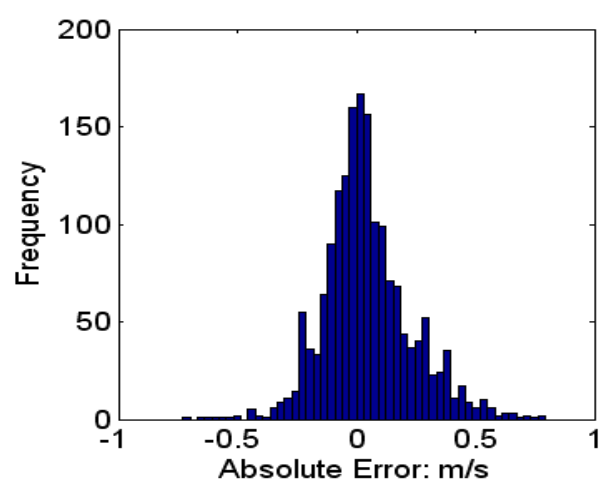

(g)

Fig. 2. Radar system OSMAR071 and data processing result: (a) The hardware structure; (b) Sea echo of range frequency Doppler spectra (c) First-order spectra determination; (d) Direction estimation at Doppler frequency $0.2815 \mathrm{~Hz}$; (e) Direction estimation at Doppler frequency $-0.2755 \mathrm{~Hz}$; (f) The ocean gravity wave phase velocity extracted from HFSWR; (g) Absolute error statistic diagram. 
Table I. Inversion result

\begin{tabular}{|c|c|c|c|}
\hline Item & $\begin{array}{c}\text { Negative frequency } \\
(\mathrm{Hz})\end{array}$ & $\begin{array}{c}\text { Positive frequency } \\
(\mathrm{Hz})\end{array}$ & $\begin{array}{c}\text { Phase velocity } \\
(\mathrm{m} / \mathrm{s})\end{array}$ \\
\hline 1 & -0.2725 & 0.2935 & 5.4321 \\
\hline 2 & -0.2635 & 0.2995 & 5.4034 \\
\hline 3 & -0.2755 & 0.2905 & 5.4321 \\
\hline 4 & -0.2606 & 0.3055 & 5.4321 \\
\hline
\end{tabular}

Fast Flourier Transform (FFT) process is used by every pulse-repetitioninterval (PRI) which called as a snapshot to produce a Range spectrum. After coherent accumulation at the same range, a second FFT is used to produce range frequency Doppler Spectrum. Fig. 2 (b) shows the measured range frequency Doppler Spectrum of 1024 PRI coherent accumulation, which is from Longhai NO.3 receiving antenna. From Fig. 2 (b), we can see that a typical HFSWR sea echoes' range frequency Doppler Spectrum contains two nearly symmetrically located first-order peaks (within $200 \mathrm{~km}$ ), second-order continuum spectral points (within $100 \mathrm{~km}$ ), the obvious distance belt distributed ionosphere reflection echo (near $150 \mathrm{~km}$ and $220 \mathrm{~km}$ ) and the noise floor.

\subsection{Result}

The analysis data is collected from July 28 to July 29, 2008. In Fig. 2 (c), the area between red lines is first-order spectra and the green line is the Bragg frequency at deep water condition. The data is at 10 range cell which is $50 \mathrm{~km}$ away from the radar site and the time is $4: 30$, July 28,2008 . The negative first-order spectra frequency is from $-0.3025 \mathrm{~Hz}$ to $-0.2546 \mathrm{~Hz}$ and the positive is from $0.2725 \mathrm{~Hz}$ to $0.3055 \mathrm{~Hz}$ respectively, the frequency resolution is $0.0030 \mathrm{~Hz}$. The signal number and direction of every first-order spectra is estimated by MUSIC algorithm. In Fig. 2 (d) the analyzed spectra Doppler frequency is $0.2815 \mathrm{~Hz}$, the estimated signal number is 2 and the signal directions are $-50^{\circ}$ and $34^{\circ}$. In Fig. 2 (e) the analyzed spectra Doppler frequency is $-0.2755 \mathrm{~Hz}$, the estimated signal number is 1 and the signal directions is $47^{\circ}$.

The ocean gravity wave phase velocity extracted by the method involved in this paper are listed in the Table I. The data is at 8 range cell which is $40 \mathrm{~km}$ away from the Longhai radar site and the time is 12:00, July 28, 2008.

\subsection{Analysis}

The radio frequency of HFSWR data collected above is $7.81 \mathrm{MHz}$, the radio wavelength is $38 \mathrm{~m}$ and the Bragg ocean gravity wave length is $19 \mathrm{~m}$. The $40 \mathrm{~km}$ away from Longhai Radar Site is deep water, so the Bragg frequency is $0.2851 \mathrm{~Hz}$, and the theory ocean gravity wave phase velocity is $5.4715 \mathrm{~m} / \mathrm{s}$. Compare with the inversion phase velocity, the error is within $0.1 \mathrm{~m} / \mathrm{s}$.

In order to confirm the accuracy of gravity wave phase velocity, the 271 range frequency Doppler spectrum from July 28 to July 29, 2008 is analyzed. The analyzed data is from range cell 6 to 10, and the distance is $30 \mathrm{~km}$ 
to $50 \mathrm{~km}$. 1726 pairs of positive and negative first-order Doppler spectra are obtained. The Fig. 2 (f) shows the inversion ocean gravity wave phase velocity. The Fig. $2(\mathrm{~g})$ shows the statistic absolute error. The confidence interval of different absolute error is that $90 \%$ inversion phase velocity's error is with threshold $0.3342 \mathrm{~m} / \mathrm{s}$, and at $85 \%, 80 \%, 75 \%, 70 \%$ the threshold are $0.2693 \mathrm{~m} / \mathrm{s}, 0.2193 \mathrm{~m} / \mathrm{s}, 0.1905 \mathrm{~m} / \mathrm{s}, 0.1618 \mathrm{~m} / \mathrm{s}$, respectively.

\section{Concluson}

The ocean gravity wave phase velocity can be extracted from HFSWR sea echo. We propound an algorithm for the ocean gravity wave phase velocity from broad beam HFSWR. The inversion phase velocity is agree well with theory calculation value by deep water sea echo analysis. The method involved in this paper is suitable for any sea water depth, moreover for the bistatic HFSWR.

\section{Acknowledgments}

This research was supported by the Twelfth-Five-Year Chinese High-Technology 863 Plan Project under grant 2012AA091701, the National Scientific Fund Committee (NSFC) under grant 60571065 and the Fundamental Research Fund for the Central Universities. The authors wish to express their gratitude to the editor and the anonymous reviews. 